\title{
II. Beiträge zur Kenntnis des 1,5-Dioxynaphtalins.
}

\section{Unter Mitbearbeitung von Constanze Bauer.}

Über das 1,5-Dioxynaphtalin ist bisher schon ziemlich viel gearbeitet worden, weil dieser Körper bequem zugänglich ist. $\left.{ }^{1}\right)$ Dabei hatte sich ergeben, daB trotz der gleichartigen Bindung der Hydroxylgruppen bei vielen Reaktionen, z. B. bei der Einwirkung von salpetriger Säure, bei der Kuppelung zu Azofarbstoffen, mit Vorliebe die Substituenten nur einmal in das Molekül eintreten. Um diese Verhältnisse näher zu klären, wurden die folgenden Versuche angestellt.

Was zunächst die Reindarstellung des 1,5-Dioxynaphtalins anbetrifft, so haben wir dabei ein anderes Verfahren eingeschlagen, als bisher in der Literatur angegeben. Wir suspendierten $40 \mathrm{~g}$ des rohen, ziemlich stark verunreinigten technischen Produktes in $1 \frac{1}{2}$ Liter Wasser, fügten $50 \mathrm{ccm}$ Alkohol hinzu und leiteten einen kräftigen Wasserdampfstrom ein. Wenn die braune Flüssigkeit längere Zeit im Kochen war, setzten wir schweflige Säure zu und kochten noch ein paar Minuten weiter. Darauf wurde rasch durch ein Faltenfilter gegossen, worauf durch Abkühlen die Substanz in schönen, nabezu farblosen Nadeln rom Schmp. $258^{\circ}$ in einer Ausbeute von 20-22 g rein erhalten wurde. Die Substanz ist gegen Alkali an der Luft sehr empfindlich und färbt sich auch durch kohlensaure Alkalien rasch intensiv rot. Zur Kontrolle der Reinheit stellten wir die Diacetylverbindung her, die wir aus verdünntem Alkohol in farblosen Blättchen vom Schmp. $159^{\circ}$ erhielten (wie in der Literatur).

1,5-Dichloracetyldioxynaphtalin. Löst man das 1,5.Dioxynaphtalin in eiskalter, verdünnter Natronlauge und gibt unter kräftigem Schütteln überschüssiges Chloracetylchlorid

1) Cleve, Bull. soc. chim. 24, 513; 26, 540; Armstrong, Ber. 20, 938; Erdmann, Ann. Chem. 247, 356; D.R.P. 41934; DRP. A. 4029; Bucherer u. Uhlmann, Chem. Centr. 1909. II, S. 1748; Bernthsen u. Semper, Ber. 20, 938; Willstätter, Ber. 47, 2796; Bentley, Robinson u. Weizmann, Chem. Centr, 1907, I, 1120; H. Kauff. mann u. Beisswanger, Ber. 36, 569 (1903); D.R.P. 68809. 
$\mathrm{zu}$, so scheidet sich eine bräunlichgefärbte Verbindung, die aus Ligroin (Tierkohle) lange weiße Nadeln ergab, die mit Petroläther gewaschen wurden.

$0,1598 \mathrm{~g}$ gabeu $0,1478 \mathrm{~g} \mathrm{AgCl}$.

Berechnet für $\mathrm{C}_{14} \mathrm{H}_{10} \mathrm{O}_{4} \mathrm{Cl}_{2}$ : $\mathrm{Cl}$ 22,66

Gefunden: $22,90 \%$.

Dibenzoyl-1,5-Dioxynaphtalin. Diese Substanz wurde in Pyridinlösung mit überschüssigem Benzoylchlorid in üblicher Weise gewonnen. Man erhält mit Wasser dann ein rötlichgelbes, dickes Öl, das von der Brühe getrennt, mehrmals mit Wasser gewaschen wurde. Setzte man Alkohol zu, so erstarrte die Masse zu gelblichen Nadeln. Aus Pyridin umkrystallisiert, gewann man die Nadeln farblos rom Schmp. 235 . Die Substanz ist unlöslich in kalter Natronlauge, schwer löslich in Alkohol, Äther, Ligroin, leichter in Toluol, Eisessig und Pyridin.

$0,1430 \mathrm{~g}$ gaben $0,4094 \mathrm{~g} \mathrm{CO}_{2}$ und $0,0578 \mathrm{~g} \mathrm{H}_{2} \mathrm{O}$.

$\begin{array}{ccc}\text { Berechnet für } \mathrm{C}_{24} \mathrm{H}_{16} \mathrm{O}_{4}: & \text { Gefunden: } \\ \mathrm{C} & 78,26 & 78,08 \% \\ \mathrm{H} & 4,34 & 4,52 \%\end{array}$

1,5-Dimethoxynaphtalin. Dieses ist schon in der Literatur ${ }^{1}$ ) beschrieben. Der Schmelzpunkt wird sehr verschieden angegeben von $174^{\circ}-175^{\circ}$ bis $183^{\circ}-184^{\circ}$. Wir fanden ihn nach öfterem Umkrystallisieren aus verdünntem Alkohol konstant bei $181^{\circ}-182^{\circ}$. Die Reinheit wurde durch Analyse kontrolliert. In konz. $\mathrm{H}_{2} \mathrm{SO}_{4}$ gelb löslich.

$0,1314 \mathrm{~g}$ gaben $0,3685 \mathrm{~g} \mathrm{CO}_{2}$ und $0,0763 \mathrm{~g} \mathrm{H}_{2} \mathrm{O}$.

Berechnet für $\mathrm{C}_{12} \mathrm{H}_{12} \mathrm{O}_{2}$ :

$\begin{array}{ccc}\mathrm{C} & 76,6 & 76,5 \% \\ \mathrm{H} & 6,4 & 6,49 \%\end{array}$

1,5-Diäthoxynaphtalin. Man löst 1,5-Dioxynaphtalin in $2 \mathrm{Mol}$. verdünnter Kalilauge, rührt und läßt langsam Diäthylsulfat zutropfen, erhitzt später gelinde bis saure Reaktion eingetreten ist, gibt dann nochmals etwas Kalilauge und Diäthylsulfat unter Erwärmen zu. Der abgeschiedene rohe Diäthyläther wird zur Entfernung von etwas Monomethyläther mit verdünnter Natronlauge warm ausgezogen und aus verdünntem Alkohol bei Gegenwart von etwas Tierkohle um-

1) Ber. 36, 569 und Chem. Centr. 1907, I, S. 1120. 
krystallisiert. Die dabei erhaltenen farblosen Blättchen schmolzen bei $130^{\circ}$. Der Körper löst sich leicht in Benzol, Toluol, Ligroin, Äther, Pyridin und starkem Alkohol, sehr schwer in Wasser.

$0,1206 \mathrm{~g}$ gaben $0,3430 \mathrm{~g} \mathrm{CO}_{2}$ and $0,0828 \mathrm{~g} \mathrm{H}_{2} \mathrm{O}$.

$\begin{array}{ccc}\text { Berechnet für } \mathrm{C}_{14} \mathrm{H}_{16} \mathrm{O}_{2}: & \text { Gefunden: } \\ \mathrm{C} & 77,7 & 77,57 \% \\ \mathrm{H} & 7,40 & 7,68 \% .\end{array}$

Durch Einwirkung von Brom auf das 1,5-Dimethoxynaphtalin in Eisessiglösung werden verschiedene Bromsubstitutionsprodukte erhalten, deren Trennung nicht leicht ist. Ein einheitliches Monobromderivat gewannen wir in folgender Weise:

$0,9 \mathrm{~g}$ Substanz wurden zunächst in heißem Benzol gelöst, dann, auf Zimmerwärme abgekühlt, mit $0,8 \mathrm{~g}$ Brom, das mit etwas Benzol verdünnt war, langsam versetzt. Die Benzollösung färbt sich bald rot unter Entwicklung ron Bromwasserstoff. Man lieB etwa $1 / 2$ Stunde lang stehen, destillierte dann das Benzol bis auf einen kleinen Rest $a b$ und versetzte mit Petroläther, wobei die Bromverbindung als gelbliche krystalline Masse gefällt wurde. Man krystallisierte aus verdünntem Alkohol bei Gegenwart von Tierkohle um und erhielt glänzende, farblose Blättchen vom Schmp. $115^{\circ}$, die sich leicht in Alkohol, Benzol, Ligroin und Pyridin lösen, schwer in kaltem Petroläther.

$0,1304 \mathrm{~g}$ gaben $0,0915 \mathrm{~g} \mathrm{AgBr}$.
Berechnet für $\mathrm{C}_{12} \mathrm{H}_{11} \mathrm{O}_{\mathbf{2}} \mathrm{Br}$ :
$\mathrm{Br}$
29,9
Gefunden: $29,86 \%$.

5-Methoxy-1-naphtol (Monomethyläther des 1,5-Dioxynaphtaiins). Dieser Äther entsteht bekanntlich ${ }^{1}$ ) neben dem Dimethyläther beim Behandeln des 1,5-Dioxynaphtalins in alkalischer Lösung mit Methylsulfat. Selbst wenn das 1,5-Dioxynaphtalin in $2 \mathrm{Mol}$. $\mathrm{KOH}$ gelöst der Einwirkung von Methylsulfat zuerst in der Kälte dann in Wärme bei $50^{\circ}-60^{\circ}$ ausgesetzt wird, erhält man etwa $1 / 3$ an Monomethyläther. Nimmt man nur $1 \mathrm{Mol}$. $\mathrm{KOH}$, so bleibt viel Dioxynaphtalin

1) Chem. Centr. 1.907, I, S. 1120. 
unangegriffen, weil sich auch dann Dimethyläther in nicht unbeträchtlicher Menge bildet. Wir arbeiteten daher mit $1 \frac{1}{2}$ Mol. KOH. Die warme Suspension ron 1,5-Dioxynaphtalin wurde unter Schütteln rasch mit $1 \frac{1}{2}$ Mol. KOH versetzt bis alles gelöst war, dann sofort abgekühlt und am Rührer mit etwas mehr als der berechneten Menge Dimethylsulfat versetzt und nach $1 / 4$ stündigem Rühren auf $50^{\circ}-60^{\circ}$ erhitzt. Die ausgeschiedene graue Masse wurde noch warm mit stark verdünnter Natronlauge mehrere Male ausgezogen. Die alkalische bräunlich gefärbte Brühe lieB man in kalte verdünnte Salzsäure einlaufen und krystallisierte den abgeschiedenen grau gefärbten Niederschlag aus Ligroin oder verdünnter Essigsäure oder auch aus Wasser. Die abgeschiedenen langen, farblosen Nadeln zeigten den Schmp. $140^{\circ}$. Der Monomethyläther ist bedeutend weniger empfindlich als das Dioxynaphtalin, während sich dieses beim Erwärmen mit Sodalösung rasch intensir rot färbt, nimmt der Monomethyläther dabei nur eine schwache Färbung an.

5-Methoxy-1-acetylnaphtol. Dieses wurde durch Erhitzen des Monomethyläthers mit überschüssigem Acetanhydrid, Ausfällen mit Wasser und Umkrystallisieren aus verdünntem Alkohol in schönen farblosen, derben Krystallen vom Schmp. $68^{\circ}$ erhalten. Die Substanz ist in den meisten üblichen Lösungsmitteln leicht löslich, sehr schwer in Wasser und in kalter, verdünnter Natroulauge.

$0,1197 \mathrm{~g}$ gaben $0,3165 \mathrm{~g} \mathrm{CO}_{2}$ und $0,0594 \mathrm{~g} \mathrm{H}_{2} \mathrm{O}$.

Berechnet für $\mathrm{C}_{13} \mathrm{H}_{12} \mathrm{O}_{3}$ :

$\mathrm{C} \quad \mathbf{7 2 , 2}$

$\mathrm{H} \quad 5,5$

Gefunden:

$72,1 \%$

5,5,

5-Methoxy-1-chloracetylnaphtol. Man lieb zur eisgekühlten Lösung des Methoxynaphtols in Natronlauge vorsichtig Chloracetylchlorid einlaufen. Die abgeschiedenen bräunlichen Flocken wurden aus sehr verdünntem Alkohol (Tierkohle) in farblosen Nadeln vom Schmp. $85^{\circ}$ erhalten, die sich leicht in Eisessig, Benzol, Toluol lösten, schwer in Ligroin und noch schwerer in Petroläther.

$0,1138 \mathrm{~g}$ gaben $0,0652 \mathrm{~g} \mathrm{AgCl}$.

Berechnet für $\mathrm{C}_{13} \mathrm{H}_{11} \mathrm{O}_{3} \mathrm{Cl}$ :

$\mathrm{Cl} \quad 14,15$

Gefunden: $14,17 \%$. 
5 -Methoxy-1-benzoylnaphtol. Dieses erhielt man durch Eintropfen von Benzoylchlorid in die Pyridinlösung des Monomethyläthers, wobei sich die Masse rötlich färbt. Mit Wasser fiel ein gelbes Öl, das beim Reiben auf Zusatz von etwas Alkohol fest erhalten wurde. Aus verdünntem Alkohol oder verdünntem Pyridin gewann man lange, silberglänzende Nadeln rom Schmp. $102^{\circ}$, die sich leicht in absolutem Alkohol, Benzol und Toluol lösten, sehr schwer in Wasser.

$0,1274 \mathrm{~g}$ gaben $0,3635 \mathrm{~g} \mathrm{CO}_{2}$ und $0,0601 \mathrm{~g} \mathrm{H}_{2} \mathrm{O}$.

Berechnet für $\mathrm{C}_{18} \mathrm{H}_{14} \mathrm{O}_{3}$ :

$\begin{array}{ccc}\mathrm{C} & 77,7 & 77,8 \% \\ \mathrm{H} & 5,0 & 5,2, .\end{array}$

5-Methoxy-2-nitroso-1-naphtol (5-Methoxy-1,2-naphtochinonoxim). 1 Mol. Monomethyläther wurde in 80 prozent. Essigsäure gelöst und gut gekühlt mit 1 Mol. Natriumnitrit in wenig Wasser langsam versetzt unter kräftigem Schütteln. Man gibt ab und zu kleine Stückchen Eis hinzu und läBt $1 / 2$ Stunde lang stehen, wobei die braun gewordene Lösung den gröBten Teil des gebildeten Nitrosokörpers in braungelben Flocken abscheidet - der Rest fällt auf Zusatz von Wasser aus. Die mit Wasser gut ausgewaschene Substanz wurde in verdünnter Sodalösung aufgenommen, von einer Verunreinigung abfiltriert und das braungelbe Filtrat mit verdünnter Salzsäure gefällt. Die hierbei abgeschiedenen dunkelgelben Flocken extrahierte man mit $\ddot{A}$ ther, trocknete den Auszug mit $\mathrm{Na}_{2} \mathrm{SO}_{4}$ und destillierte den Äther bis auf einen kleinen Rest ab. Dann schied sich auf Zusatz von etwas Petroläther und $A b-$ kühlen durch Eis die Nitrosoverbindung in schönen dunkelgelben Nadeln $a b$, die beim Erhitzen bei ca. $165^{\circ}$ unter Zersetzung schmolzen. Die alkalische Lösung der Substanz ist braungelb, die in Alkohol, Pyridin, Äther, Eisessig gelb. In Ligroin ist der Körper schwer löslich.

$0,1029 \mathrm{~g}$ gaben $0,2456 \mathrm{~g} \mathrm{CO}_{2}$ und $0,0435 \mathrm{~g} \mathrm{H}_{2} \mathrm{O}$.

$0,1068 \mathrm{~g}$ gaben $6,5 \mathrm{~cm} \mathrm{~N}$ bei $18^{\circ}$ und $744 \mathrm{~mm}$.

Berechnet für $\mathrm{C}_{11} \mathrm{H}_{9} \mathrm{O}_{9} \mathrm{~N}$ :

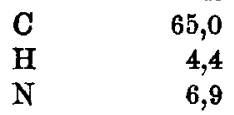

Gefunden:

$\begin{array}{rr}\mathrm{C} & 65,0 \\ \mathrm{H} & 4,4 \\ \mathrm{~N} & 6,9\end{array}$

$65,1 \%$

4,7,

7,0 " .

Journal f. prakt. Chemie [2] Bd. 94. 
Bei der Einwirkung von salpetriger Säure auf $\alpha$-Naphtol entstehen bekanntlich zwei Nitrosonaphtole, in unserem Falle war die Suche nach einem Isomeren vergeblich.

5-Methoxy-2-amino-1-naphtol. Man löste $1 \mathrm{~g}$ des Nitrosokörpers in $40 \mathrm{~g}$ thiophenfreiem, scharf getrocknetem Benzol, erhitzte zum Sieden und fügte nun tropfenweise reines Phenylhydrazin zu. Es beginnt alsbald lebhafte Gasentwicklung; wenn diese nachläBt, wird noch einige Zeit weiter erhitzt, bis die braune Farbe der Lösung rötlich geworden ist. Nun kühlt man rasch im Schälchen auf Eis ab, worauf sich ca. $0,8 \mathrm{~g}$ fast farblose Blättchen abscheiden, die rasch abgesaugt und mit trockenem Petroläther gewaschen wurden. Aus reinem trockenen Benzol umkrystallisiert, gewann man farblose, silberglänzende Blättchen vom Schmp. $158^{\circ}$ unter Zersetzung. Die Substanz ist im feuchten Zustande sehr empfindlich und färbt sich dann grün. Mit verdünntem Eisenchlorid färbt sich die Lösung in Salzsäure intensir rot und scheidet bei Zugabe ron mehr Eisenchlorid rotbraune Flocken ab. Beim Schütteln der Base in Ammoniak an der Luft tritt Grünfärbung ein.

$0,1186 \mathrm{~g}$ gaben $7,6 \mathrm{ccm} \mathrm{N}$ bei $20^{\circ}$ und $745 \mathrm{~mm}$.

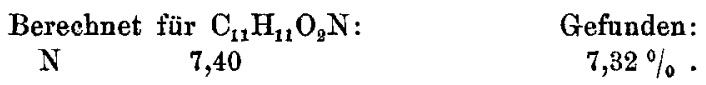

5-Methoxy-1,2-naphtalindioxim. 4 g 5-Methoxy-2nitroso-1-naphtol wurden mit $10 \mathrm{~g}$ Wasser übergossen, $4 \mathrm{~g}$ salzsaures Hydroxylamin zugesetzt und nun mit etwa $100 \mathrm{ccm}$ Methylalkohol, dem man einige Tropfen konzentrierte Salzsäure zugesetzt hatte, gemischt und etwa 10 Stunden lang am RückfluBkühler gekocht. Die anfangs braungelbe Lösung wird nach und nach dunkelrot. Beim Erkalten schieden sich ca. 4,1 g, zum Teil aus langen, braunroten Nadeln bestehend, eines Gemisches zweier Substanzen aus, die durch verdünnte Natronlauge getrennt wurden, wobei das Dioxim mit citronengelber Farbe in Lösung geht, während der alkaliunlösliche Körper dessen Anhydrid darstellt. Das aus der alkalischen Lösung mit Essigsäure gefällte Dioxim $(2,2 \mathrm{~g})$ wurde aus wenig heißer Essigsäure in schönen goldgelben, zu Sternchen oder Warzen vereinigten Nadeln erhalten, die sich bei $195^{\circ}$ zersetzten. Die 
Verbindung ist in Alkohol und Äther ziemlich leicht löslich, schwerer in Benzol. In konzentrierter Schwefelsäure löst sie sich dunkelviolett bis blauschwarz, beim Verdünnen mit Wasser zunächst rot werdend, bei stärkerem Verdünnen fällt die Substanz in gelben Flocken wieder aus.

$0,1132 \mathrm{~g}$ gaben $0,2507 \mathrm{~g} \mathrm{CO}$, und $0,0475 \mathrm{~g} \mathrm{H}_{\mathrm{g}} \mathrm{O}$.

$0,1083 \mathrm{~g}$ gaben $12,3 \mathrm{ccm} \mathrm{N}$ bel $19^{\circ}$ und $739 \mathrm{~mm}$.

Berechnet für $\mathrm{C}_{11} \mathrm{H}_{10} \mathrm{O}_{5} \mathrm{~N}_{2}$ :

$\begin{array}{lcc}\mathrm{C} & 60,5 & 60,4 \% \\ \mathrm{H} & 4,6 & 4,7 \% \\ \mathrm{~N} & 12,8 & 12,9 \%\end{array}$

Beim spontanen Erhitzen, z. B. auf Platinblech, verpufft der Körper.

Wie oben bemerkt entsteht bei der Einwirkung von Hydroxylamin auch das in verdünnten Alkalien unlösliche Anhydrid, 5-Methoxy-1,2-naphtalindioximanhydrid (5-Methoxy-1,2naphtofurazan). Das Rohprodukt wurde in wenig heiBem Eisessig gelöst, mit Tierkohle behandelt und dann der heiBen filtrierten Lösung so viel Wasser zugesetzt, bis eine Trübung entstand. Es krystallisierten dann beim Abkühlen lange feine, schwach gelb gefärbte Nadeln aus, die bei $159^{\circ}$ schmolzen. Die Nadeln lösen sich leicht in Eisessig, Alkohol, Toluol, schwer in Ligroin, Petroläther und kaltem Äther. Von konzentrierter Schwefelsäure werden sie gelbrot aufgenommen, beim Verdünnen mit Wasser das Anhydrid wieder in weiBen Nädelchen abscheidend.

$0,1250 \mathrm{~g}$ gaben $0,3016 \mathrm{~g} \mathrm{CO}$ und $0,0478 \mathrm{~g} \mathrm{H}_{\mathrm{g}} \mathrm{O}$.

$0,1107 \mathrm{~g}$ gaben $14,0 \mathrm{~cm} \mathrm{~N}$ bei $23^{\circ}$ und $734 \mathrm{~mm}$.

$\begin{array}{ccc}\text { Berechnet für } \mathrm{C}_{11} \mathrm{H}_{8} \mathrm{O}_{2} \mathrm{~N}_{2}: & \text { Gefunden: } \\ \mathrm{C} & 66,0 & 65,8 \% \\ \mathrm{H} & 4,0 & 4,2 \% \\ \mathrm{~N} & 14,0 & 14,1 \%\end{array}$

Durch die Bildung dieses Anhydrids ist bewiesen, daB im Dioxim die Oximreste die 1,2-Stellung einnehmen, so daB daher auch das 5-Methoxynitrosonaphtol die angenommene Konstitution haben muB, wofür auch die intensive Färbang der Nitrosoverbindung spricht.

1,5-Monomethyldioxynaphtalin als Azokomponente. Beim Kuppeln des Monomethyläthers in alkalischer Lösung werden sowohl Mono- wie auch Bisazofarbstoffe gebildet. 
$1,7 \mathrm{~g}$ wurde in $20 \mathrm{ccm} 20$ prozent. Natronlauge gelöst und $250 \mathrm{ccm}$ Eiswasser zugesetzt, dann lieB man die Diazoniumchloridlösung aus $0,9 \mathrm{~g}$ Anilin langsam unter Rühren einlaufen. Dabei scheidet sich nur eine geringe Menge des in Alkali unlöslichen Bisazofarbstoffs rom Schmp. $204^{\circ} \mathrm{ab}$, den man abfiltrierte. Das erste Filtrat wurde mit Essigsäure heiB gefällt und aus verdünntem Alkohol in roten Nadeln mit grünlichem Obertlächenschimmer gewonnen. Der Farbstoff schmilat bei $155^{\circ}$ unter Zersetzung, löst sich in stark verdünnter Natronlauge lachsfarben, in konzentrierter Schwefelsäure stahlblau, in Alkohol gelbrot.

$0,1115 \mathrm{~g}$ gaben $9,8 \mathrm{~cm} \mathrm{~N}$ bei $17^{\circ}$ und $743 \mathrm{~mm}$.

Berechnet für $\mathrm{C}_{17} \mathrm{H}_{14} \mathrm{~N}_{2} \mathrm{O}_{2}$ :

N $\quad 10,07$

Gefunden:

$10,11 \%$.

Durch Kochen des Farbstoffs mit 5-6 Teilen Acetanhydrid gewinnt man die Acetylverbindung, die aus verdünntem Alkohol in gelbbraunen Warzen krystallisiert, die bei $123^{\circ}$ schmelzen.

$0,1137 \mathrm{~g}$ gaben $0,2979 \mathrm{~g} \mathrm{CO}_{2}$ und $0,0528 \mathrm{~g} \mathrm{H}_{2} \mathrm{O}$.

Berechnet für $\mathrm{C}_{19} \mathrm{H}_{18} \mathrm{~N}_{2} \mathrm{O}_{3}$ :

C $\quad \mathbf{7 1 , 2 5}$

$\mathrm{H} \quad 5,0$

Gefunden:
$71,45 \%$
$5,2 \%$

Der Azofarbstoff ist demnach als 5-Methoxy-4-benzolazo-1-naphtol zu bezeichnen.

Bisaz ofarbst off (5-Methoxy-2, 4-bisbenzolazo-1-naphtol). Durch den Eintritt eines zweiten Diazoniumradikals in das Molekül des Monoazofarbstoffs wird ein alkaliunlöslicher Bisazofarbstoff erzeugt, der demnach in der Hydrazonform vorliegt. Daraus folgt, daB die zweite Benzolazogruppe in die 2-Stellung des Moleküls eintritt. In die dauernd stark alkalische gehaltene Lösung von 1 Mol. 5-Methoxy-1-naphtol läBt man unter guter Kühlung und Rühren 2 Mol. Benzoldiazoniumchlorid einlaufen. Es scheiden sich dunkelrote Flocken ab, die man absaugt und einige Male mit lauwarmer verdünnter Natronlauge auszieht, um noch kleine Mengen des Monoazofarbstoffs zu entfernen; dann wird mit Wasser ausgewaschen und der Bisazofarbstoff aus verdünntem Pyridin umkrystallisiert, wobei schöne bronzefarbene, lange Nadeln vom Schmp. $204^{0}$ iunter 
O. Fischer u. Bauer: 1,5-Dioxynaphtalin. 21

Zersetzung) erhalten wurden. Die Substanz ist in Alkohol mit tiefroter Farbe löslich; von konzentrierter Schwefelsäure wird sie mit hellgrüner Farbe aufgenommen.

$0.1110 \mathrm{~g}$ gaben $14,3 \mathrm{ccm} \mathrm{N}$ bei $19^{\circ}$ und $743 \mathrm{~mm}$.

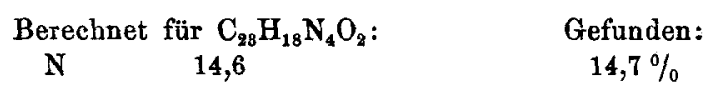

Einwirkung von salpetriger Säure auf 1,5-Dioxynaphtalin.

Nitrosodioxynaphtaline sind bereits in der Patentliteratur angegeben, aber nicht genauer beschrieben. Hier kam es darauf an festzustellen, ob das 1,5-Dioxynaphtalin nur e in Mononitrosoprodukt oder auch ein Bisnitrosoderivat gebe. Wir haben daher unter verschiedenen Bedingungen 1 und $2 \mathrm{Mol}$. salpetriger Säure einwirken lassen, dabei immer nur einen Mononitrosokörper erhalten. Dieser dürfte, da er stark gefärbt ist und weil bei der Einwirkung von salpetriger Säure auf 1,5-Dioxynaphtalinmonomethyläther die Nitrosogruppe in die 2-Stellung tritt, 2-Nitroso-1,5-dioxynaphtalin sein. Zur Darstellung verfuhren wir so, daB wir das Dioxynaphtalin (1 Mol.) entweder in 70 prozent. Essigsäure gut gekühlt mit 1 oder 2 Mol. Natriumnitrit versetzen, oder in verdünnter alkoholischer Lösung bei Gegenwart von Essigsäure oder Salzsäure arbeiteten. In allen Fällen schied sich von selbst oder durch Zusatz von Eisstücken ein bräunlichroter Niederschlag ab, der aus sehr verdünntem Alkohol schöne rotbraune Nadeln ergab, die sich bei ca. $190^{\circ}$ zersetzten. In verdünnter Natronlauge oder auch in kohlensaurem Alkali löst sich die Substanz mit purpurroter Farbe, in Alkohol braunrot und in konzentrierter Schwefelsäure violett. Die Nitrosoverbindung ist leicht löslich in Alkohol, Holzgeist, Eisessig, Pyridin, etwas weniger leicht in $\ddot{A}$ ther und heißem Wasser, schwer in Ligroin und Petroläther.

$0,1254 \mathrm{~g}$ gaben $0,2919 \mathrm{~g} \mathrm{CO}_{2}$ und $0,0425 \mathrm{~g} \mathrm{H}_{2} \mathrm{O}$.

$0,1204 \mathrm{~g}$ gaben $8,0 \mathrm{ccm} \mathrm{N}$ bei $24^{\circ}$ and $733 \mathrm{~mm}$.

$\begin{array}{ccc}\text { Berechnet für } \mathrm{C}_{10} \mathrm{H}_{7} \mathrm{O}_{8} \mathrm{~N}: & \text { Gefunden: } \\ \mathrm{C} & 63,50 & 63,49 \% \\ \mathrm{H} & 3,70 & 3,79 \% \\ \mathrm{~N} & 7,40 & 7,37, .\end{array}$ 
1,5-Dioxynaphtalin als Azokomponente. Das 1.5Dioxynaphtalin bildet, mit Diazoniumsalzen gekuppelt, mit Vorliebe Monoazofarbstoffe, wie dies auch die bisherigen Erfahrungen ergeben haben. ${ }^{1}$ ) Wir kuppelten in üblicher Weise sowohl in alkalischer Lösung mit oder ohne Zusatz von Pyridin, wie auch in verdünnter alkoholischer Lösung bei Gegenwart von Salzsäure oder Natriumacetat. Immer wurde der Hauptsache nach ein und derselbe Monoazofarbstoff gebildet, während sich selbst bei Anwendung von $2 \mathrm{Mol}$. Diazoniumsalz nur wenig eines Polyazofarbstoffs bildete. So entstand mit Benzoldiazoniumchlorid 4-Benzolazo-1,5-dioxynaphtalin, das sich in Alkalien mit purpurroter Farbe, in konzentrierter Schwefelsäure blaugrün, beim Verdünnen mit Wasser zunächst flaschengrün färbte, dann violett und rot werdend. Der Farbstoff wurde durch Lösen in verdünntem Alkali und Abfiltrieren einer kleinen Menge eines unlöslichen Körpers (Polyazofarbstoff) und Wiederausfällen mit Essigsäure in roten Flocken abgeschieden, die, in heißem Eisessig gelöst, auf Zusatz ron etwas Wasser kleine rote Nadeln vom Schmp. $210^{\circ}$ (unter Zersetzung) ergaben. Der Farbstoff löst sich leicht in Pyridin und in Eisessig, schwerer in Alkohol mit blutroter Farbe, die durch Zusatz von konzentrierter Salzsäure zunächst violett und dann bei weiterem Zusatz blaugrün wird.

$0,1154 \mathrm{~g}$ gaben $10,9 \mathrm{ecm} \mathrm{N}$ bei $17^{\circ}$ und $728 \mathrm{~mm}$.

Berechnet für $\mathrm{C}_{16} \mathrm{H}_{12} \mathrm{~N}_{2} \mathrm{O}_{2}$ : $\mathrm{N} \quad 10,6$

Gefunden: $10,65 \%$.

4-p-Nitrobenzolazo-1,5-dioxynaphtalin. 1,6 g Dioxynaphtalin wurden in $60 \mathrm{ccm} 20$ prozent. Natronlauge gelöst und mit $40 \mathrm{ccm}$ Pyridin und $200 \mathrm{~cm}$ Wasser versetzt. Man lieb nun die aus $1,38 \mathrm{~g}$ p-Nitranilin bereitete Diazoniumlösung langsam unter Rühren einlaufen, indem durch Eis gut gekühlt wurde. Die Masse färbt sich alsbald blau; nach $1 / 2$ stündigem Rühren wurde die alkalische Flüssigkeit von einer geringen Menge eines unlöslichen, grünlich gefärbten Körpers abfiltriert und mit Essigsäure der dunkelrote Farbstoff gefällt. Dieser löst sich in konzentrierter Schwefelsäure mit hellgrüner Farbe, die auf Zusatz von Wasser braunrot wird. Der Azokörper wurde durch Umkrystallisieren aus verdünntem Pyridin in

1) Vgl. Willstätter u. Wheeler, Ber. 47, 2799 (1914). 
O. Fischer u. Bauer: 1,5-Dioxynaphtalin.

roten, grüı glänzenden, derben Krystallen erhalten, die bei ca. $250^{\circ}$ schmelzen und sich zersetzen.

$0,1286 \mathrm{~g}$ gaben $15,6 \mathrm{~cm} \mathrm{~N}$ bei $18^{\circ}$ and $726 \mathrm{~mm}$.

Berechnet für $\mathrm{C}_{16} \mathrm{H}_{11} \mathrm{~N}_{3} \mathrm{O}_{4}$ :

N $\quad 13,6$

Gefunden:

$13,6 \%$.

Nach den Erfahrungen beim Kuppeln des 5-Methoxy1-naphtols war zu erwarten, daB auch das 1,5-Dioxynaphtalin unter gewissen Bedingungen Polyazofarbstoffe geben werde. Theoretisch wären hier zwei Bisazo-, ein Trisazo-, und sogar ein Tetrakisazokörper denkbar. Tatsächlich erhielten wir jedoch nur einen Bisazofarbstoff, obschon wir in stark alkalischer Lösung mit 3 und 4 Mol. Benzoldiazoniumchlorid kuppelten. $1,6 \mathrm{~g}$ Dioxynaphtalin wurde in $6 \mathrm{Mol}$. $\mathrm{NaOH}$ gelöst und durch Hineinwerfen von Eisstücken gut gekühlt. Wir ließen nun unter lebhaftem Rühren zunächst 2, dann nochmals 2 Mol. der Diazoniumlösung einlaufen. Es schied sich schon bei der ersten Operation ein tiefroter, in wäBrigem Alkali unlöslicher Körper ab, der abgesaugt und aus Pyridin schöne, cantharidenglänzende Nadeln von einem sehr hohen Schmelzpunkt (über $\left.250^{\circ}\right)$ abschied. Diese Substanz ist ein Bisazofarbstoff.

$0,1272 \mathrm{~g}$ gaben $17,4 \mathrm{ccm} \mathrm{N}$ bei $21^{\circ}$ und $736 \mathrm{~mm}$.

Berechnet für $\mathrm{C}_{22} \mathrm{H}_{18} \mathrm{~N}_{4} \mathrm{O}_{2}$ : $\mathrm{N} \quad 15,2$

Gefunden: $15,4 \%$.

Der Farbstoff löst sich schwer in Alkohol rotbraun, sowie auch in Benzol, Ligroin, Methylalkohol, in Pyridin mit tiefroter Farbe ziemlich leicht löslich. In konzentrierter Schwefelsäure löst or sich malachitgrün, beim Verdünnen mit Wasser wieder rot werdend. Auffällig ist die Unlöslichkeit in wäBrigen Alkalien, jedoch zeigte sich, daB der Farbstoff von alkoholischem Kali leicht mit tiefvioletter Farbe aufgenommen wird. Es war daher möglich, daB diese Unlöslichkeit in wäBrigem Alkali den Eintritt weiterer Azogruppen bei der Kuppelung hinderte. Deshalb wurde der Bisazofarbstoff nochmals in alkoholischer Lösung bei Gegenwart von viel Natronlauge mit Benzoldiazoniumchlorid zusammengerührt. Die Farbe blieb jedoch unverändert tiefviolett und der mit Wasser abgeschiedene Körper war identisch mit dem Bisazofarbstoff. Unter den geschilderten Umständen bildet sich also kein höherer Polyazofarbstoff. 